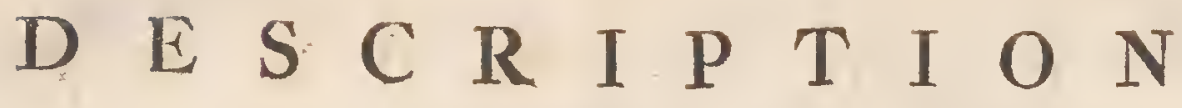
D'UNE BELLE ESPËCE DE

\section{PERROQUET DES INDES,}

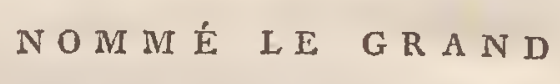

\section{LORY ROUGE-POURPRÉ,}

\author{
Qui se trouve à \\ C E Y L A N,
}

Et que l'on conferve dans le CABINET de

SON ALTESSE SÉRÉNISSIME

MONSEIGNEUR LE PRINCE D'ORANGE ET DE NASSAU, $S T A D H O U D E R$ HER REDITAIRE, GOUVERNEUR, CAPIT'AINE GE'NE'RAL ETAMIRAL DES PROVINCES-UNIES

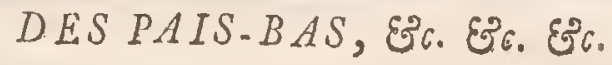
$\begin{array}{llll}P & A & R\end{array}$
A. $\quad \mathrm{V} \quad \mathrm{O} \quad \mathrm{S} A \mathrm{~A} R$,

Direcieur des Cabinets d'Hiftoire Naturelle छo de Currofités de S. A. S., Membre de P.Académie Impériale, Correppondant de l'Acadénie Royale des Sciences de Paris, E Mombre de la Socièté des Sciences de Zélande à Vilfingue.

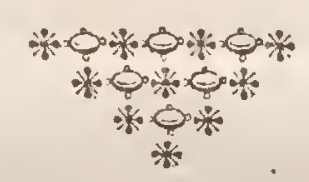

$A$ A $M$ M $S$ S T

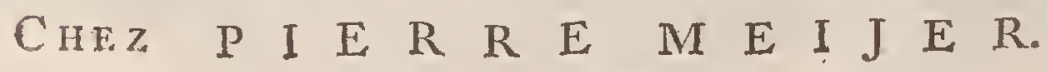
M. D C C. L X I X. 


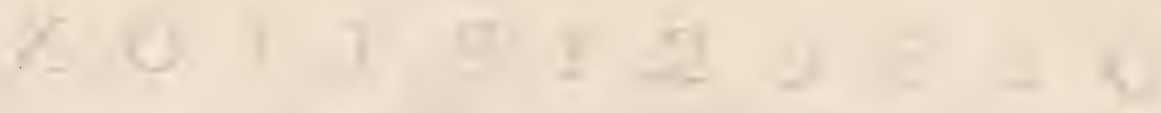

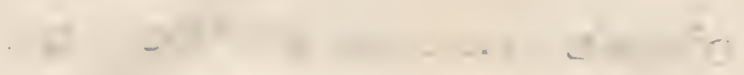

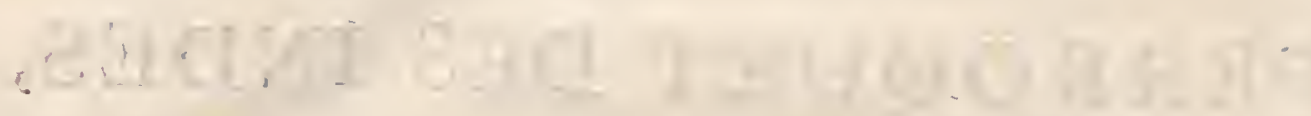

$$
\begin{aligned}
& \text { - }
\end{aligned}
$$

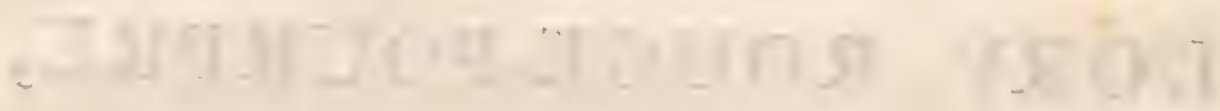
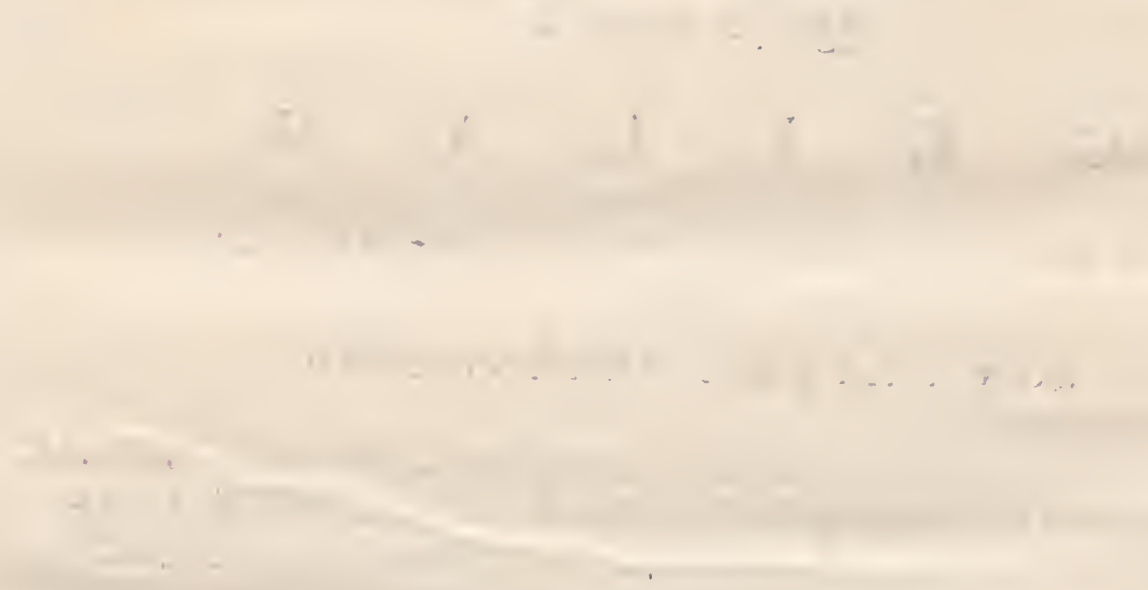

$+$

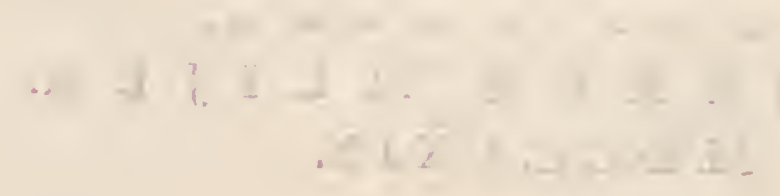




\section{HISTOIRE NATURELLE}

\section{U G R A N D \\ LORY ROUGE-POURPRÉ, \\ D E C E Y L A N.}

$\mathrm{O}^{\mathrm{n}}$

$\mathrm{N}$ diroit, en voyant un Auteur (a) nous donner quatrevingt-quinze variétés de Perroquets, que ce nombre doit renfermer toutes les Efpeces qui compofent ce Genre. Mais rier n'eft moins vrai, tant le graind Auteur de Ia Nature paroît illimité dans la diverfité admirable de fes créatures.

Le charmant Oiteau, qui fe préfente ici , \& qu'on peut, à jufte titre, mettre au rang des plus beaux de toute la Famille des Perroquets, n’a point encorè été décrit que je fache, \& il manque tant à la Lifte de Mr. LiN BrisSON. Nos Géographes étrangers, qui, pour ta plupart, ont traité fort fuperficiellement l'Hiftoire Naturelle, ou fe font copiés les uns les autres, n'en font pas non plus la moindre mention.

(a) Le laborieux BrissoN a rapporté ce nombre d'Efpèces différentes, fous le. quel on doit néanmoins comprendre toutes les variétés du Genre; telles que le grand Perroquet \& longue queue, auquel nous donbons le nom impropre de Raff (Corbeau); le petit à longue queue, que nous appellons Parkiet-Ranfje, (petit Corbeal-Perroquet), qui font tous connus fous la dénomination générale de Perro. qutets. Ceux qu'on appelle Lory's, ne font diftingués des autres que par leur couleur orlinaire, qui eft un rouge, plus ou moins beau \& éclatant chez l'un que chez l'autre; mais ce caractere ne fuffit point pour les féparer des Perroquets pro. prement dits. Enfuite viennent ceux 'qu'ou a nommés Kakatous, reconnoiffables à la belle hupe dont ils font ornés; Et enfin ceux qu'on appelle Parakietjes ou MofcbParkietjes, (petits Pcroquets. Moineaux) affez connus par leur peticefre. Peutetre s'en trourc.t.il, entre les variétćs de Mr. Brisson, quelques-uns qui ne différent que par le fexe; Ceci, joint à l'incertitude oil l'on eft fouvent fi les Oifraux qu'on a devant foi ont achęé o: aon leur crue, forme de très grandes difficultés dans la détermination de lears Efpèces. Ornirbolog. Tom. IV. pag. 182.

A 2 


\section{* DESCRIPTIONDUGRAND}

Il y a quelques années que SON ALTESSE SÉRÉNISSIME, fe trouvant à fa Maifon de Plaifance au Bois, achetta celui-ci d'un Homme reyenu des Indes, qui difoit l'avoir apporté de CeỹIn. Cette jolie bête ne vécut à la Cour que peu dé mois. Quelque magnifique \& ravifant que fut fon plumage, fon naturel n’y répondoit pas ; "contre lordinaire des Lorys, qui font fort dociles (b), celui-ci ne parloit point, \& ne vouloit rien appren. dre; d'ailleurs il n'étoit pas méchant, \& c'étoit ce qu'on pou. voit dire de mieụx de lui, après avoir été ébloui de fes brillantes couleurs, étourdi de fes cris aigus. On mit un jour, auprès

(b) SEza décrit un Lory, des Ines des Papous, lequel parloit diftinetement cinq Cangues différentes. - 1 e matin il chantoic un perit air en préfence de fon Mâ̂tre, à qui il étojt fort attaché; après quoi il crioit: goeden morsen Sinjeur leen Wyn fopje (bon jour, Monfieur! ma mouillette au vin) ee qu'il réputioit aufi le foir, \& fiffluic entre deux." Quand il ne voyruir pas' fon Mâ̂rre, il s'attriftuit, \& ne vouloit ni manger ni parler. Son Mâtre lé vendit pour cing cent florins; mais la Siléliré de cet Oifrap étoit, telle, qu'il mourut peu de jours après. SEв A Tuefourus
Tonn I. Tab. 38. Fig. 4.

Enwarus dans ion Hift. Nat. des Oifeaux, Tom. IV. Tab. ipo. repréfente un petit Lory. Celui.ci fut envoyé vivant, l'année palfée, à SON ALTESSE SÉRóntssime, par Monfieur Hope Repréfentant de ce Prince, aupès de la Cimpagnie des Indes Orientales. Il vient de Bengale, \&. c'eft le plus docile, le plus familicr \& le plus joli petit animal (car il n'eft pas grand) que l'on puiffe, voir. Il prononce très diftinctement différens mots, \& fifie fort bien; il fe couche fur lo dos, prend de fon bec \& de fes pattes, la main qu'on lui préfente pour jouer, \& étant mis à terre, il court après les gens, en fautillant comme un Moincau. II danfe fur fon juchoir, \& fur les doigts dc celui qui le tient, criant Lory, Lory, d'une voix clairc \& agréable. Ce charmant petit animal fait les délices de SON Azteśst Royale Madame la PRINCESSE D'ORARGe ET DE NAsSaU, \&ce. \&c. \& c. Les couleurs de cet Oifean font beaucoup plus bellcs \& plus uives que Mr. EDWAR DS ne les repréfente. Peu de jours après cette defcription il eff
venu à mourir.

Le Cabinet du Prince, entre pliffeurs autres, en offre encore un, que Mon. fieur E D W ARD S Tub. 169 . repiéfente parfaitement bien, \& qui par fa forme, mais von par les couleurs paroît. etre un petit Lory; quiqu'd dire vrai je ne puife pas décousrir de différences affez ferfibles-pour diftinguer les Lorys des Perroquets propiement dits. Ce joli petit animal étoit auffi d'une gentillefre, dont, a l'exception du pécédent, je ne connois point d'exemple. J'ajoute ceci, unique. ment pour faire voir que ces Ef-èces de Perroquets en particulier font fort doci. les, d'un naturel doux, \& gu'on les apprivoife facilement. 
près de lui un Moineau, pour voir ce qu'il en feroit; l'événement fut funefte au pauvre animal; il le tua d'un coup de bec, mais il n'en mangea que la cervelle.

Les Perroquets, ì parler généralement, font dociles \& amis de l'Homme. Les Auteurs en racontent des merveilles, indépendamment de ce qui peut paroître fabuleux. J'ai vu moimème, it Rotterdam, un Perroquet accompagner de fou chant fa Maîtreffe, qui jouoit du Clavecin, \& exécuter, avec elle, divers Airs d'une façon admirable.

Leurs alimens ordinaires font toutes fortes de fruits d'Arbres, L'adreffe \& la force qu'ont ces Oifeaux dans leur bec, femblent au premier conp d'œil indiquer, que ce bec eft approprié à brifer des corps qui offrent plus de réfiftance que les tendres fruits des Arbres; auffi leur fert-il non feulement ì fe défendre contre divers animaux carnaciers, qui grimpent de nuit fur les Arbres dans ces Contrées-lì ; mais encore principalement à caffer les noyaux de certains fruits qu'ils mangent volontiers, \& dont quelques. uns font revêtus d'une coque très dure. Ajoutez à cela que le bec des Perroquets lour tient lieu d'ur troifième pied pour monter.

Suivant LaBAT (c) ils font leurs Nids dans les Arbres creux, ou entre des branches, \& en couvrent le fond de quelques-unes de leurs plumes. Le favant Mr. VALMONT DE BomaEr (d) parle aufie d'un Nid fort fingulier, qu'ils conftruifent de petits rameaux, \& le fufpendent à l'extrêmité des plus hautes branches des Arbres, fans doute pour mieux garantir leur couvée. Ce récit s'accorde avec la Defcription \& la Figure que SE B A (e) donne d'un femblable Nid, qui fe voit dans le Cabi-

net

(c) Nieurwe Reize naar de Franfcbe Eilanden van America. C. \&. d. Nouveaus Poyages aux llies Francoifes de l'Amérique. i, Part. pug. 272. Amft. 1725. 4\%:

(d) Dictionnaire d'Hilt. Nut. Tom. Il. pag. 235. Paris 1764. $8^{\circ}$.

(e) Thefaurus. Vob 1. pag. 10+. Tab.68 Fig. 1..

A 3 
net du Prince. Selon la plupart des Ecrivains, les Perroquets ne pondent que deux Oeufs tachetés, à-peu-près de la groffeur de ceux de Pigeon, \& qui font couvés tour-it-tour par le Mâle \& la Femelle. Le même LABAT l'apporte, que. l'an í707, il y avoit, à Paris, chez la Veuve de Mr. A u ge R., Gouverneur de St. Domingue, deux Perroquets, qui avoient eu plufieurs fois des petits, mais qu'ils ne reftoient pas en vie, vraifemblablement fiute de noumiture propre ì de fi jeunes Oifeaux. J'ai vu fouvent en ce Pays ici un petit œuf, mais tout blanc, qu'un Perroquet pondoit cinque année dans fa cage.

Les Perroquets peuvent devenir fort vieux; \& j’en comois un qui cft depuis près d'un fiècle dans certaine Famille. Cependint ils font fujets à l'Epilepfie, fur-tout les Lorys. L'e meilleur remède à ce mal eft de mêler leur pain bien trempé, avec une bonne quantité de chenevi en poudre. Les Lorys Indiens requie: rent en général une nourriture plus humectée, que n'eft celle des Perroquets de l'Amérique. Il eft auffi aftez fingulier que la fe: mence du Cotonier enyvre, ces Oifeaux, \& qu'ils ne foient point incommodés de l'ufage du Saffían-bitțard, qui eft un fi violent purgatif pour les Hommes $(f)$.

$\therefore$ Ce que PIST ORJus $(g)$ rapporte eft remarquable, \& temble prouver que les Perroguets, quoiqu'ils propagent à Surinam, y font aufi Oifeaux de palfage. "Aux mois d'Aloût \& de Sep"tembre des années 1750 \& $175 \mathrm{I}$. (dit-il) tems auquel on "2. fait la récolte du Caffe, l'on vir, à Surinam, une prodigieure „- quantité de Pcrioquets de toutes fortes, qui fondoient en trou"pes fur le Caffé, dont ils détachoient \& mangeoient la capfule ;rouge, \& rejettoient à tẹre les fèves. L’an I 760 vers : 1e $\therefore .1$ , mê-

(f) Houttuin Nat. Hifl. of uitvoerige Befcbryving der Dieren, enz. C. ̀े. d. Hijt. Nut out ample Defcription des. Animaux, Plantes E Minéraux fuivant le Syftème de Mr.LIN as us. I. Part. Cb.IV. p. 233. Amft. I762. $8^{\circ}$.

(g.) Befcbryving van de Colonie van Surinamen. C. à. d. Defoription de la Colopir \&e Surinana, Pag. 08 . Amjt. $1763.14 \%$ 


\section{LORY ROUGE-POURPRE' DE CEYLAN;}

¡. même tems, on vit de nouveau d'auffi nombreux effains de

"Perroquets, qui s'étendirent tout le long de la Còte, \& y fi"rent un dégât affreux, fans qu'on aìt pù découvrir d'où ve"noient ces animaux en fi grand nombre ". Le même Auteur \& d'autres difent auffi, que dans les narines des jeunes Perroquets, \& dans une tumeur qu'ils ont fur la tête, il s'y engendre un Ver court \& gros, qui tombe au bout de quelque tems, \& qu'enfuite le trou qu'il a occupé fe referme $(b)$.

Il y a différentes façons de prendre ces Oifeaux. Ceux qu'on veut garder en vie pour leur apprendre à parler, font tirés fort jeunes de leurs Nids, au mois d'Août, fuivant PISTORIUs.

Au Bréfil, on abbat les vieux de deflus les Arbres, avec de très Iongues fléches, dont la pointe eft bien.enveloppée de cotton, pour qu'elle ne les bleffe pas.

Lават dit que les Caraïbes mettent de nuit des charbons autour des $\Lambda$ rbres fur lefquels fe tiennent les Perroquets, \& qu'ils jettent là-deffus une Comme avec du Piment verd, dont la fumée étourdit ces Oifeaux, \& les fait tomber à terre devant eux. VAN BRAKEL (i) nous apprend que les Indiens fur la Rivicie

(b) Il faut furement'que cela foit occafionné par quclque Infecte de l'Efpèce des Mouches, ou de celle des Guêpes, qui choififent ces endroits pour y dépofer \& faire éclorre leurs œufs; ce qui arrive de mêne aux Rennes, \&c. Cette réflexion me rappelle le Pouxarlé (Gevleugelde-Luis) comme $\mathrm{Mr}$ S L A в R. R le nomme \& le décrit dans les Diflertations de la Snciété Hollandoife des Sciences, X. Part Cb. II. pag. 4I3. Au premier afpeet de la Figure, je fus furpris d'abord de la dénonination de Pou, fans me fouvenir de la Defcripion que le célèbre Mr. de REAUMUR en avoit déja donnée fous le nom de Mouche, Que j'examinc la ftruclure de cet Infecte, fes ailes, \& principalement fa longue trompe applatie au bout avec un rehord; que je parcoure les Deferiptions de Meflieurs de REA UMUR \& SLABBER, je trouve plutôt une Mouche qu'un Pou, qui confie fes œufs \& fes petits aux Hyrondelles. Je lis aufi, ainfi que Mr. SLA BnER, chez. Mr. de REAUMur Tom. IV. Mém. 3. qu'il avoit trouvé les Nids des Hyrondelles remplis de Puces, \& de Vers de Puces, ou de Puces en leur premiere forme: pent-Ĉrre ceux-ci font-ils les Puccs des Hyrondelles.

(i) Americaanjebe Voyagen, pag. 85. Amft. 1695. 4\%. 
de Berbice, les prennent avec des lacets attachés à des båton's, qu'ils jettent à la tête des Oifeaux.

La chair de ces Perroquets eft une fort bonne nourriture. LA. BAT, qui dit avoir mangé plus de Perroquets en Amérique, que de Perdrix en Europe, l'affirme en ajoutant que la chair des jeunes eft délicate \& fort fucculente, foit étuvée, foit rôtie à la broche. ou dans des feuilles de vigne. Les vieux Oifeaux donnent une bonne foupe; mais leur chair prend l'odeur \& le goût des alimens dont ils font ufage; \& de-lì vient qu'ils font fouvent très bons, \& fouvent auffi très mauvais à manger.

La faculté d'articuler des mots, fuculté qui diftingue ces animaux de tous les autres, les rend fort remarquables. Mais comme la diffection n'entre proprement pas dans notre Plan, \& que nous manquons entre autres fecours, d'un Anatomifte aufi judicicux \& aufí habile que Mr. de BufF on en a trouvé un dans la perfonne de Mr. DAUBENTON, nous renvoyons à cet égard le Lećteur curieux à l'Ouvrage du favant HouT TuYN, qui a 'rapporté, dans fon Hiftoire Naturelle $(k)$, tout ce qu'il a trouvé de relatif à cet objet, après une infinité de recherches.

(k) Natuurlyke Hiftorie der Dieren, enz. I. Deel IV: Siuk Pag. 22.

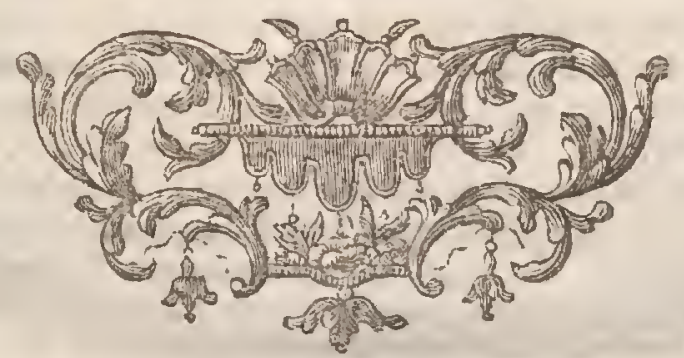

DES. 
5筷

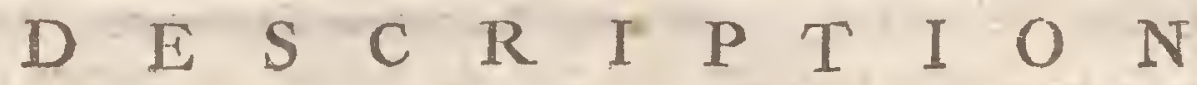

D U G R A N D

\section{LORY ROUGE-POURPRÉ, D E C E Y L A N. Tab. V I I.}

T A grandeur de cet Oifeau furpaffe un peu celle du Per* roquet commun de couleur plombée (Eritbacus LiNN.) \& eft furpafrée à fon tour par celle de la double Amazone, (Affivus LINN.)

La tête entiere, le col, une partie de la poitrine, font d'un bel écarlatte foncé, tirant fur le pourpré; \& tels font auffi le dos, la plus grande partie des ailes, des plumes des pieds, \& les parties inferieures près de la queue; de forte que felon la couleur dominante l'on peut nommer cet Difeau le Lory rouge - pourpré. La poitrine feule eft parée d'un bleu terni, comme il fe voit aux Prunes bleues, dont la rofée n'eft point encore effacée, \& où éclate une teinte de rouge-pourpré.

Ce même bleu de la poitrine qui defcend jufques fur les jambes, s'élève des deux côtés vers le fouet des ailes, \& fait le tour du bas du col, en s'étendant vers le dos. Les fouets des ailes font bordés de petites plumes d'un très beau bleu; le bout des groffes plumes, \& même la moitié extérieure des premières ou des plus grandes, font d'un beau bleu foncé. Les plumes de la queue font rouges en deflus, \& leurs bouts d'un très beau jaune clair. En deffous les mêmes plumes font proprement tout-ì- 
fait jaunes, mais au milieu elles ont une teinte de rouge jaunâtre clair.

Le bec de ces Oifeaux eft noir; les narines font petites, ovales, \& ne fortent que tant foit peu de defrous les plumes du front.

Les yeux font noirs, avec un cercle jaune. La langue ..... Les jambes font d'un 'gris-noir, de même que les ferres, qui ont deux doigts tournés en avant \& deux en arriere, dont l'intérieur eft le plus court. Les ongles font grandes, crochues, noires \& pointuës.

\section{F 1 N.}

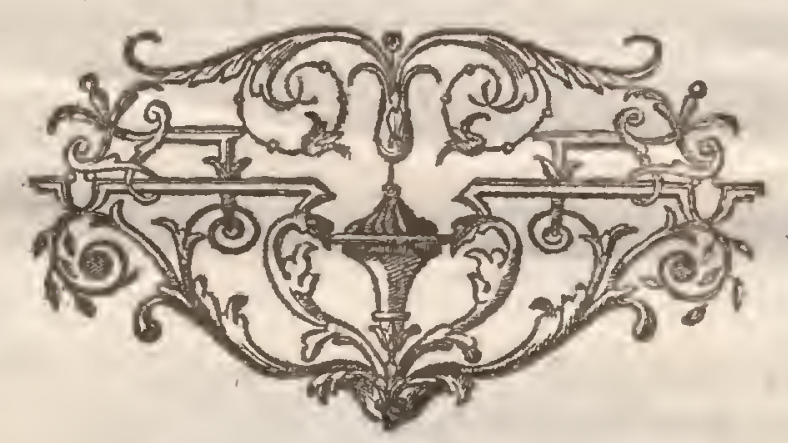






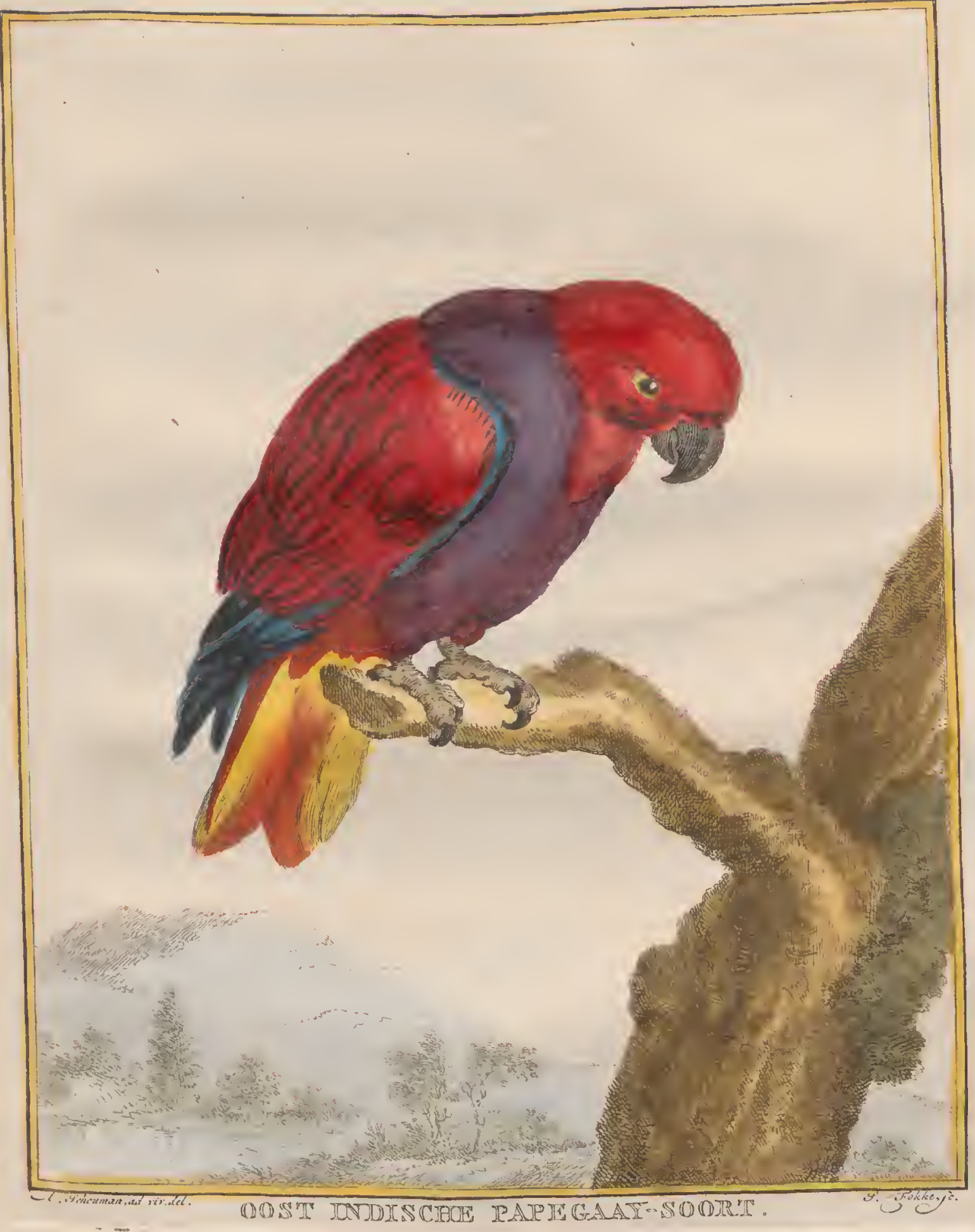


